\title{
A Switched Approach to Robust Stabilization of Multiple Coupled Networked Control Systems
}

\author{
Mei Yu, ${ }^{1}$ Nan Ding, ${ }^{2}$ Wen Tan, ${ }^{1}$ and Junyan $Y u^{3}$ \\ ${ }^{1}$ School of Control and Computer Engineering, North China Electric Power University, Beijing 102206, China \\ ${ }^{2}$ State Nuclear Electric Power Planning Design and Research Institute, Beijing 102206, China \\ ${ }^{3}$ School of Mathematical Sciences, University of Electronic Science and Technology of China, Chengdu, Sichuan 611731, China
}

Correspondence should be addressed to Mei Yu; yumei@pku.org.cn

Received 19 March 2014; Accepted 8 May 2014; Published 11 June 2014

Academic Editor: Bo Shen

Copyright (C) 2014 Mei Yu et al. This is an open access article distributed under the Creative Commons Attribution License, which permits unrestricted use, distribution, and reproduction in any medium, provided the original work is properly cited.

\begin{abstract}
This paper proposes a switched approach to robust stabilization of a collection of coupled networked controlled systems (NCSs) with node devices acting over a limited communication channel. We suppose that the state information of every subsystem is split into different packets and only one packet of the subsystem can be transmitted at a time. Multiple NCSs with norm-bounded parameter uncertainties and multiple transmissions are modeled as a periodic switched system in this paper. State feedback controllers can be constructed in terms of linear matrix inequalities. A numerical example is given to show that a collection of uncertain NCSs with the problem of limited communication can be effectively stabilized via the designed controller.
\end{abstract}

\section{Introduction}

Networked control systems (NCSs) are feedback control systems with network communication channels used for the communications between spatially distributed system components like sensors, actuators, and controllers. In recent years, the studies of NCSs have received increasing attention in control theory [1-4]. The use of the communication channels can reduce the costs of cables and power, simplify the installation and maintenance of the whole system, and increase the reliability. The insertion of communication network in the feedback control loop complicates the analysis and design of an NCS because many ideal assumptions made in the traditional control theory cannot be applied to NCSs directly. The traditional control theory requires that all the feedback information be obtained by the controller. Meanwhile one major problem must be solved, the limited bandwidth of the communication network. Sometimes, data packets containing full plant measurements may not be permitted to be transmitted because of limited bandwidth and this may deteriorate the system performance and destabilize the system. Examples include fleets of unmanned autonomous vehicles, wide area power system, remote planetary exploration with multiple coordinated robots, and the control of microactuator arrays. In such systems, simultaneous communication with all subsystems may not be possible because of physical or performance constraints. So how to control the NCSs via a limited communication channel is a big problem. Potential application can be found in [5-7].

The problem of stabilization with finite communication bandwidth has received much attention; see, for example, [2, 8-11]. In NCSs, communication capacity depends on the topology of the network. In many cases, the communication among the devices of the network is through one communication channel as shown in Figure 1, where $P_{i}$ is the plant and $C_{i}$ is the corresponding controller $(i=1, \ldots, N)$. The problem of stabilization with finite communication bandwidth was introduced by $[12,13]$. The switched system approach was introduced to study NCSs with multiple-packet transmission in $[14,15]$ and further pursued by $[16,17]$. The merit of the switched approach is that the controllers can make full use of the previous information to stabilize NCSs when the current state measurements are available from the network. By switched approach, the stabilization 


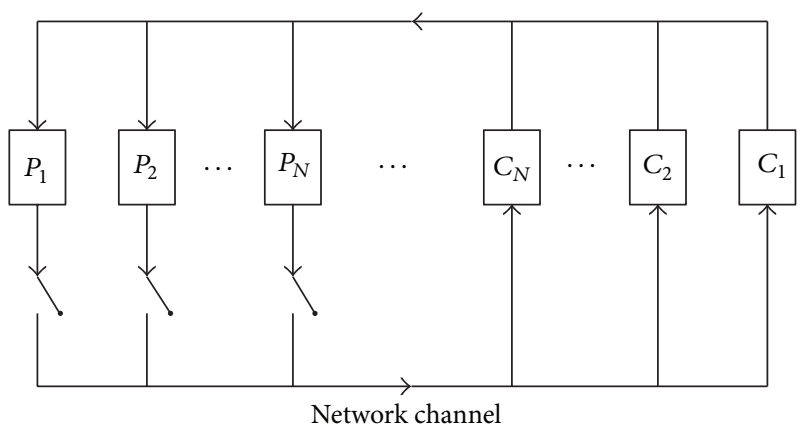

Figure 1: A shared network.

of NCSs with multiple-packet transmissions over tokenpassing bus networks was considered in $\mathrm{Yu}$ et al. [14, 15], where sufficient conditions for stabilization and design of controllers have been obtained through modeling NCSs as periodically switched systems. Further results for single NCS were presented in $\mathrm{Yu}$ et al. [18-21]. Zhang and HristuVarsakelis [22] also studied stabilization problem of NCSs by modeling these systems as switched systems. Reference [23] studied the stability for a class of systems with time varying delay subject to controller failure, where the delay system with failed controller was modelled as a class of switched delay systems. Unfortunately, all the above literatures focused on studying NCSs without uncertainty.

Uncertainty is ubiquitous in control systems and it inevitably exists in system model due to the complexity of the system itself, exogenous disturbance, measurement errors, and so on. Qiu et al. [24] dealt with mode and parameter-dependent robust mixed $\mathrm{H}_{2} / \mathrm{H}_{\infty}$ filtering design for a class of discrete-time switched polytopic linear systems. Furthermore, the problem of delay-dependent robust energyto-peak filtering design for a class of discrete-time switched linear systems with a time-varying state delay and polytopic uncertainties was revisited in [25]. Recently, [26] studied exponential $H_{\infty}$ filtering for discrete-time switched statedelay systems under asynchronous switching. Reference [27] considered the problem of robust stabilization of linear uncertain discrete-time systems via limited capacity communication channels. In [28], a class of networked control systems was investigated where the plant had time-varying norm-bounded parameter uncertainties. In [29], sufficient conditions were given to ensure the stability of uncertain NCSs using switched approach. Reference [30] studied the stability of NCSs that were subject to time-varying transmission intervals, time-varying transmission delays, and communication constraints. Reference [31] addressed the problem of stabilizing uncertain nonlinear plants over a shared limited bandwidth packet-switching network. However, all the above literatures consider performance or stabilization of single NCS, which was not coupled with any other system.

$\mathrm{Yu}$ et al. [32] investigated stabilization of a collection of linear systems with limited information. Reference [16] further modeled multiple NCSs as a periodic switched system, with only one subsystem able to access the network to transmit all of its present state information at a time.
But they did not deal with the problem of multiple packet transmission. Ding et al. [33] studied multiple networked control systems with multiple transmissions, but they did not consider uncertainties and they did not consider the twoside channel transmission. In [34, 35], Dai et al. developed a scheduling strategy for a collection of discrete-time NCSs subjected to communication constraints, which were modelled as a switched delay system. The systems they considered were not coupled.

To the best of our knowledge, there is no result on the robust stabilization of a collection of coupled NCSs with multiple transmissions. Motivated by the references above, this paper will model multiple coupled NCSs with normbounded parameter uncertainties as switched system and then we can apply the theory of the switched system to NCSs [36, 37]. We consider the case that all the nodes act over a limited bandwidth communication channel, state information of every subsystem is split into different packets and only one packet can be transmitted at a time. Applying the toking bus protocol, the nodes are arranged logically into a ring and transmit their packets in a predetermined order. For multiple-packet transmitted NCSs with one-side channel and with both S/C and C/A network channel, we model such multiple coupled uncertain NCSs as a periodic switched system. We consider the setup with a clock-driven sensor, and both the controller and the actuator are event driven. The controller is installed to use the old state measurement if there is no new data updating. Then robust stability of the NCSs with periodic transmission is considered. State feedback controllers can be constructed in terms of linear matrix inequalities (LMIs).

The remainder of this paper is structured as follows. Section 2 models multiple NCSs with multipacket transmission and norm-bounded parameter uncertainties. Section 3 develops stabilization and stability results for state feedback case. Section 4 presents a numerical simulation to illustrate the efficiency and feasibility of our proposed approach. Section 5 concludes this paper.

Notation. We use standard notations throughout this paper. Denote by $A^{T}$ the transpose of a matrix $A . A>0(A<$ 0 ) means that $A$ is positive definite (negative definite). $I$ is the identifying matrix of appropriate dimension. $R^{n}$ and $R^{n m}$ denote, respectively, the $n$ dimensional Euclidean space and the set of all $\mathrm{nm}$ real matrices. In symmetric block matrices, the symbol $*$ is used as an ellipsis for terms induced by symmetry. In token bus, the computers are connected so that the signal travels around the network from one computer to another in a logical ring. A single electronic token moves around the ring from one computer to the next. If a computer does not have information to transmit, it simply passes the token on to the next workstation.

\section{System Modelling}

We consider a finite collection of linear systems that are coupled together through their dynamics and feedback. We consider the case that the state information is transmitted 


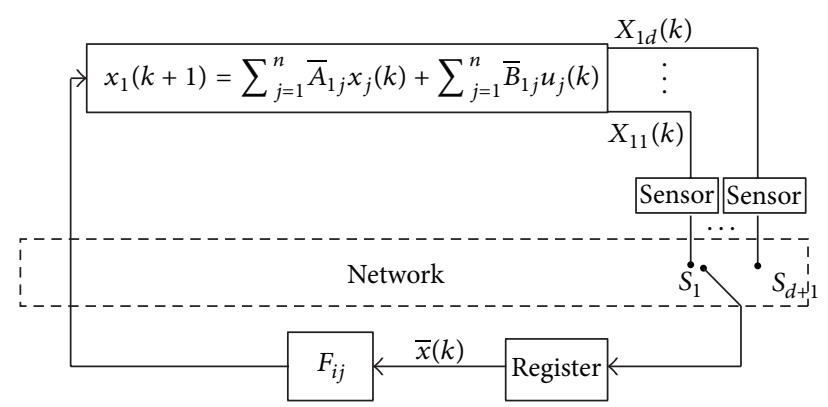

FIGURE 2: The structure of an NCS with S/C channel.

over a limited bandwidth communication channel. State information of every subsystem is split into different packets and only one packet can be transmitted at a time. We first consider multiple-packet transmission NCSs with only S/C channel, illustrated in Figure 2. The $n$ coupled NCSs with norm-bounded parameter uncertainties are described by

$$
\begin{gathered}
x_{i}(k+1)=\sum_{j=1}^{n}\left(A_{i j}+\Delta A_{i j}\right) x_{j}(k)+\sum_{j=1}^{n}\left(B_{i j}+\Delta B_{i j}\right) u_{j}(k), \\
u_{i}(k)=\sum_{j=1}^{n} F_{i j} \bar{x}_{j}, \quad i, j=1,2, \ldots, n, k=1,2, \ldots,
\end{gathered}
$$

where $x_{i}(k) \in \mathbf{R}^{n}$ and $u_{i}(k) \in \mathbf{R}^{p}$ are the plant state and the plant input, respectively. $\bar{x}_{i}(k)$ is the content of the register. $F_{i j}$ is the feedback gain to be designed. $A_{i j}, B_{i j}$ are known real constant matrices with appropriate dimensions. $\Delta A_{i j}, \Delta B_{i j}$ characterize the uncertainties in the system and satisfy the following assumption:

$$
\left[\begin{array}{ll}
\Delta A_{i j} & \Delta B_{i j}
\end{array}\right]=E_{i j} \Gamma_{j}(k)\left[\begin{array}{ll}
G_{j} & H_{j}
\end{array}\right],
$$

where $E_{i j}, G_{j}$, and $H_{j}$ are known real constant matrices of appropriate dimensions, and $\Gamma_{j}(k)$ are unknown matrix functions with Lebesgue-measurable elements which satisfies

$$
\Gamma_{j}(k)^{T} \Gamma_{j}(k) \leq I, \quad \forall k
$$

Define $\bar{A}_{i j}=A_{i j}+\Delta A_{i j}, \bar{B}_{i j}=B_{i j}+\Delta B_{i j}$; then the networked control systems with norm-bounded parameter uncertainties are described as

$$
\begin{aligned}
x_{i}(k+1) & =\sum_{j=1}^{n} \bar{A}_{i j} x_{j}(k)+\sum_{j=1}^{n} \bar{B}_{i j} u_{j}(k), \\
u_{j}(k) & =\sum_{j=1}^{n} F_{i j} \bar{x}_{j}, \quad i, j=1,2, \ldots, N, k=1,2, \ldots
\end{aligned}
$$

Suppose the state is split into $d$ packets

$$
x_{i}(k)=\left[X_{i 1}^{T}(k), \ldots, X_{i d}^{T}(k)\right]^{T},
$$

where $X_{i t}(k)=\left[x_{i r_{t-1}+1}(k) \cdots x_{i r_{t}}(k)\right]^{T}$ and $1 \leq t \leq d, 0=$ $r_{0}<r_{1}<\cdots<r_{d}=n$. The controller is installed to use the old state measurement if there is no new data updating. That is,

$$
\bar{x}_{i}(k)=\left[\bar{X}_{i 1}^{T}(k), \ldots, \bar{X}_{i d}^{T}(k)\right]^{T}
$$

where

$$
\bar{X}_{i t}(k)= \begin{cases}X_{i t}(k) & \text { if the packet containing } \\ & X_{i t}(k) \text { is transmitted; } \\ \bar{X}_{i t}(k-1) & \text { otherwise. }\end{cases}
$$

2.1. An Example of Two Subsystems. For simplicity, we first consider an example of two subsystems and the plant states of each subsystem are split into two packets. Consider

$$
\begin{aligned}
& x_{1}(k+1)=\bar{A}_{11} x_{1}(k)+\bar{A}_{12} x_{2}(k)+\bar{B}_{11} u_{1}(k)+\bar{B}_{12} u_{2}(k), \\
& x_{2}(k+1)=\bar{A}_{21} x_{1}(k)+\bar{A}_{22} x_{2}(k)+\bar{B}_{21} u_{1}(k)+\bar{B}_{22} u_{2}(k),
\end{aligned}
$$

where $x_{1}(k)=\left[X_{11}^{T}(k), X_{12}^{T}(k)\right]^{T}, x_{2}(k)=\left[X_{21}^{T}(k), X_{22}^{T}(k)\right]^{T}$.

In the standard token-passing bus protocol, the token is repeatedly transmitted in a fixed order in the network, that is, $[1,1,2,2]$ in turn. Here, we regard different packets as different nodes for the network protocol, and this means the packets would be transmitted in a periodic manner via the network channel. With the given four-step communication cycle, the system equations will evolve according to

$$
\begin{aligned}
x_{1}(k+1)= & \bar{A}_{11} x_{1}(k)+\bar{A}_{12} x_{2}(k) \\
& +\bar{B}_{11} F_{11}\left[X_{11}^{T}(k), \bar{X}_{12}^{T}(k-1)\right]^{T} \\
& +\bar{B}_{12} F_{12}\left[\bar{X}_{21}^{T}(k-1), \bar{X}_{22}^{T}(k-1)\right]^{T}, \\
x_{1}(k+2)= & \bar{A}_{11} x_{1}(k+1)+\bar{A}_{12} x_{2}(k+1) \\
& +\bar{B}_{11} F_{11}\left[X_{11}^{T}(k), X_{12}^{T}(k+1)\right]^{T} \\
& +\bar{B}_{12} F_{12}\left[\bar{X}_{21}^{T}(k-1), \bar{X}_{22}^{T}(k-1)\right]^{T}, \\
& \bar{A}_{11} x_{1}(k+2)+\bar{A}_{12} x_{2}(k+2) \\
& +\bar{B}_{11} F_{11}\left[X_{11}^{T}(k), X_{12}^{T}(k+1)\right]^{T}
\end{aligned}
$$




$$
\begin{aligned}
& +\bar{B}_{12} F_{12}\left[X_{21}^{T}(k+2), \bar{X}_{22}^{T}(k-1)\right]^{T}, \\
& x_{1}(k+4)=\bar{A}_{11} x_{1}(k+3)+\bar{A}_{12} x_{2}(k+3) \\
& +\bar{B}_{11} F_{11}\left[X_{11}^{T}(k), X_{12}^{T}(k+1)\right]^{T} \\
& +\bar{B}_{12} F_{12}\left[X_{21}^{T}(k+2), X_{22}^{T}(k+3)\right]^{T}, \\
& x_{1}(k+5)=\bar{A}_{11} x_{1}(k+4)+\bar{A}_{12} x_{2}(k+4) \\
& +\bar{B}_{11} F_{11}\left[X_{11}^{T}(k+4), X_{12}^{T}(k+1)\right]^{T} \\
& +\bar{B}_{12} F_{12}\left[X_{21}^{T}(k+2), X_{22}^{T}(k+3)\right]^{T}, \\
& x_{2}(k+1)=\bar{A}_{21} x_{1}(k)+\bar{A}_{22} x_{2}(k) \\
& +\bar{B}_{21} F_{21}\left[X_{11}^{T}(k), \bar{X}_{12}^{T}(k-1)\right]^{T} \\
& +\bar{B}_{22} F_{22}\left[\bar{X}_{21}^{T}(k-1), \bar{X}_{22}^{T}(k-1)\right]^{T}, \\
& x_{2}(k+2)=\bar{A}_{21} x_{1}(k+1)+\bar{A}_{22} x_{2}(k+1) \\
& +\bar{B}_{21} F_{21}\left[X_{11}^{T}(k), X_{12}^{T}(k+1)\right]^{T} \\
& +\bar{B}_{22} F_{22}\left[\bar{X}_{21}^{T}(k-1), \bar{X}_{22}^{T}(k-1)\right]^{T}, \\
& x_{2}(k+3)=\bar{A}_{21} x_{1}(k+2)+\bar{A}_{22} x_{2}(k+2) \\
& +\bar{B}_{21} F_{21}\left[X_{11}^{T}(k), X_{12}^{T}(k+1)\right]^{T} \\
& +\bar{B}_{22} F_{22}\left[X_{21}^{T}(k+2), \bar{X}_{22}^{T}(k-1)\right]^{T}, \\
& x_{2}(k+4)=\bar{A}_{21} x_{1}(k+3)+\bar{A}_{22} x_{2}(k+3) \\
& +\bar{B}_{21} F_{21}\left[X_{11}^{T}(k), X_{12}^{T}(k+1)\right]^{T} \\
& +\bar{B}_{22} F_{22}\left[X_{21}^{T}(k+2), X_{22}^{T}(k+3)\right]^{T}, \\
& x_{2}(k+5)=\bar{A}_{21} x_{1}(k+4)+\bar{A}_{22} x_{2}(k+4) \\
& +\bar{B}_{21} F_{21}\left[X_{11}^{T}(k+4), X_{12}^{T}(k+1)\right]^{T} \\
& +\bar{B}_{22} F_{22}\left[X_{21}^{T}(k+2), X_{22}^{T}(k+3)\right]^{T} \text {. }
\end{aligned}
$$

In this case, the two subsystems $x_{1}$ and $x_{2}$ are coupled and their dynamics have a periodicity of four steps. Similar to [10], define the buffered states as

$$
\begin{aligned}
& \widehat{x}_{1}(k)=\left[\begin{array}{c}
x_{1}(k-3) \\
x_{1}(k-2) \\
x_{1}(k-1) \\
x_{1}(k)
\end{array}\right], \\
& \widehat{x}_{2}(k)=\left[\begin{array}{c}
x_{2}(k-3) \\
x_{2}(k-2) \\
x_{2}(k-1) \\
x_{2}(k)
\end{array}\right], \quad \widetilde{x}=\left[\begin{array}{c}
\widehat{x}_{1} \\
\hat{x}_{2}
\end{array}\right] .
\end{aligned}
$$

Then the corresponding drift dynamics are

$$
\begin{aligned}
& \widehat{x}_{1}(k+1)=\left[\begin{array}{cccc}
0 & I & 0 & 0 \\
0 & 0 & I & 0 \\
0 & 0 & 0 & I \\
\bar{B}_{11} F_{11} D_{10} & 0 & 0 & \bar{A}_{11}+\bar{B}_{11} F_{11} D_{11}
\end{array}\right] \widehat{x}_{1}(k) \\
& +\left[\begin{array}{cccc}
0 & 0 & 0 & 0 \\
0 & 0 & 0 & 0 \\
0 & 0 & 0 & 0 \\
0 & \bar{B}_{12} F_{12} D_{21} & \bar{B}_{12} F_{12} D_{20} & \bar{A}_{12}
\end{array}\right] \widehat{x}_{2}(k) \\
& \widehat{x}_{2}(k+1)=\left[\begin{array}{cccc}
0 & I & 0 & 0 \\
0 & 0 & I & 0 \\
0 & 0 & 0 & I \\
\bar{B}_{21} F_{21} D_{10} & 0 & 0 & \bar{A}_{21}+\bar{B}_{21} F_{21} D_{11}
\end{array}\right] \widehat{x}_{1}(k) \\
& +\left[\begin{array}{cccc}
0 & 0 & 0 & 0 \\
0 & 0 & 0 & 0 \\
0 & 0 & 0 & 0 \\
0 & \bar{B}_{22} F_{22} D_{21} & \bar{B}_{22} F_{22} D_{20} & \bar{A}_{22}
\end{array}\right] \widehat{x}_{2}(k)
\end{aligned}
$$

where

$$
\begin{array}{lll}
D_{11}=\left[\begin{array}{ll}
I & 0 \\
0 & 0
\end{array}\right], & D_{10}=\left[\begin{array}{ll}
0 & I \\
0 & 0
\end{array}\right], \\
D_{21}=\left[\begin{array}{ll}
0 & 0 \\
I & 0
\end{array}\right], & D_{20}=\left[\begin{array}{ll}
0 & 0 \\
0 & I
\end{array}\right] .
\end{array}
$$

The combined drift dynamics at each of the four steps of the communication sequence are

$$
\tilde{x}(k+1)=M_{q} \tilde{x}(k),
$$


where

$$
\begin{aligned}
M_{1} & =\left[\begin{array}{ccccccccc}
0 & I & 0 & 0 & 0 & 0 & 0 & 0 \\
0 & 0 & I & 0 & 0 & 0 & 0 & 0 \\
0 & 0 & 0 & I & 0 & 0 & 0 & 0 \\
\bar{B}_{11} F_{11} D_{10} & 0 & 0 & \bar{A}_{11}+\bar{B}_{11} F_{11} D_{11} & 0 & \bar{B}_{12} F_{12} D_{21} & \bar{B}_{12} F_{12} D_{20} & \bar{A}_{12} \\
0 & 0 & 0 & 0 & 0 & I & 0 & 0 \\
& 0 & 0 & 0 & 0 & 0 & 0 & I & 0 \\
& 0 & 0 & 0 & 0 & 0 & 0 & 0 & I \\
\bar{B}_{21} F_{21} D_{10} & 0 & 0 & \bar{A}_{21}+\bar{B}_{21} F_{21} D_{11} & 0 & \bar{B}_{22} F_{22} D_{21} & \bar{B}_{22} F_{22} D_{20} & \bar{A}_{22}
\end{array}\right], \\
M_{2} & =\left[\begin{array}{ccccccccc}
0 & I & 0 & 0 & 0 & 0 & 0 & 0 \\
0 & 0 & I & 0 & 0 & 0 & 0 & 0 \\
0 & 0 & 0 & I & 0 & 0 & 0 & 0 \\
0 & 0 & \bar{B}_{11} F_{11} D_{11} & \bar{A}_{11}+\bar{B}_{11} F_{11} D_{10} & \bar{B}_{12} F_{12} D_{21} & \bar{B}_{12} F_{12} D_{20} & 0 & \bar{A}_{12} \\
0 & 0 & 0 & 0 & 0 & I & 0 & 0 \\
0 & 0 & 0 & 0 & 0 & 0 & I & 0 \\
0 & 0 & 0 & 0 & 0 & 0 & 0 & I \\
0 & 0 & \bar{B}_{21} F_{21} D_{10} & \bar{A}_{21}+\bar{B}_{21} F_{21} D_{11} & \bar{B}_{22} F_{22} D_{21} & \bar{B}_{22} F_{22} D_{20} & 0 & \bar{A}_{22}
\end{array}\right],
\end{aligned}
$$$$
M_{3}=\left[\begin{array}{cccccccc}
0 & I & 0 & 0 & 0 & 0 & 0 & 0 \\
0 & 0 & I & 0 & 0 & 0 & 0 & 0 \\
0 & 0 & 0 & I & 0 & 0 & 0 & 0 \\
0 & \bar{B}_{11} F_{11} D_{11} & \bar{B}_{11} F_{11} D_{10} & \bar{A}_{11} & \bar{B}_{12} F_{12} D_{20} & 0 & 0 & \bar{A}_{12}+\bar{B}_{12} F_{12} D_{21} \\
0 & 0 & 0 & 0 & 0 & I & 0 & 0 \\
0 & 0 & 0 & 0 & 0 & 0 & I & 0 \\
0 & 0 & 0 & 0 & 0 & 0 & 0 & I \\
0 & \bar{B}_{21} F_{21} D_{11} & \bar{B}_{21} F_{21} D_{10} & \bar{A}_{21} & \bar{B}_{22} F_{22} D_{20} & 0 & 0 & \bar{A}_{22}+\bar{B}_{22} F_{22} D_{21}
\end{array}\right],
$$$$
M_{4}=\left[\begin{array}{cccccccc}
0 & I & 0 & 0 & 0 & 0 & 0 & 0 \\
0 & 0 & I & 0 & 0 & 0 & 0 & 0 \\
0 & 0 & 0 & I & 0 & 0 & 0 & 0 \\
\bar{B}_{11} F_{11} D_{11} & \bar{B}_{11} F_{11} D_{10} & 0 & \bar{A}_{11} & 0 & 0 & \bar{B}_{12} F_{12} D_{21} & \bar{A}_{12}+\bar{B}_{12} F_{12} D_{20} \\
0 & 0 & 0 & 0 & 0 & I & 0 & 0 \\
0 & 0 & 0 & 0 & 0 & 0 & I & 0 \\
0 & 0 & 0 & 0 & 0 & 0 & 0 & I \\
\bar{B}_{21} F_{21} D_{11} & \bar{B}_{21} F_{21} D_{10} & 0 & \bar{A}_{21} & 0 & 0 & \bar{B}_{22} F_{22} D_{21} & \bar{A}_{22}+\bar{B}_{22} F_{22} D_{20}
\end{array}\right] .
$$

Remark 1. For data packets transmitted in both C/A and S/C channels periodically, similar model can be obtained.

As shown in Figure $3, \bar{x}(k)$ is the received state information by the controller over S/C channel, $u(k)$, which will be sent to the system over C/A channel, is the output of the controller, and $\bar{u}(k)$ is the real system input. We assume that plant output and controller output are divided into $d(d \geq 1)$ packets to be transmitted. The plant input is presented as (5) and the controller output is described as

$$
u_{i}(k)=\left[\bar{U}_{i 1}^{T}(k), \ldots, \bar{U}_{i d}^{T}(k)\right]^{T},
$$

where

$$
U_{i t}(k)=\left[\begin{array}{lll}
u_{i r_{t-1}+1} & (k) \cdots & u_{i r_{t}}(k)
\end{array}\right]^{T},
$$

where $1 \leq t \leq d, 0=r_{0}<r_{1}<\cdots<r_{d}=n$.
Consequently, denote

$$
u_{i}(k)=\left[U_{i 1}^{T}(k), \ldots, U_{i d}^{T}(k)\right]^{T},
$$

where

$$
U_{i t}(k)= \begin{cases}U_{i t}(k), & \text { if the packet containing } \\ \bar{U}_{i t}(k-1), & U_{i t}(k) \text { is transmitted; }\end{cases}
$$

Then

$$
u_{i}(k)=\sum_{j=1}^{n} F_{i j}\left[\bar{X}_{i 1}^{T}(k) \cdots \bar{X}_{i d}^{T}(k)\right]^{T},
$$

where $\bar{x}_{i}(k)$ is defined in (6).

In the case that both $\mathrm{C} / \mathrm{A}$ and $\mathrm{S} / \mathrm{C}$ channels are standard token-passing bus networks, the packets in the two channels 


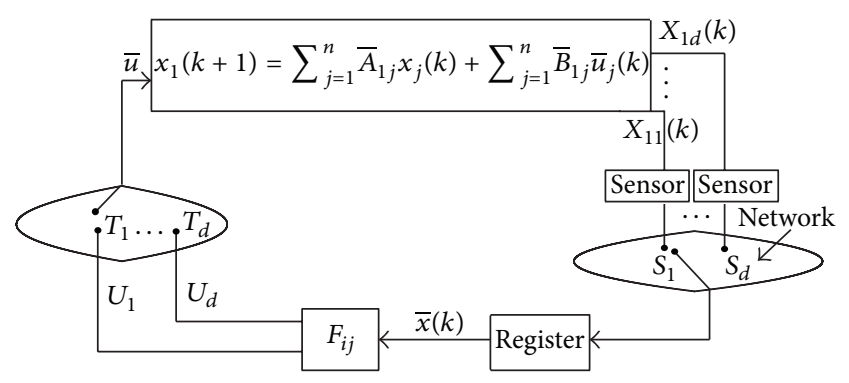

FIGURE 3: The structure of an NCS with both S/C and C/A channel.

would be transmitted synchronously in a periodic manner. We still study the following two subsystems and there is only one packet that can be transmitted every time:

$$
\begin{aligned}
& x_{1}(k+1)=A_{11} x_{1}(k)+A_{12} x_{2}(k)+B_{11} \bar{u}_{1}(k)+B_{12} \bar{u}_{2}(k), \\
& x_{2}(k+1)=A_{21} x_{1}(k)+A_{22} x_{2}(k)+B_{21} \bar{u}_{1}(k)+B_{22} \bar{u}_{2}(k),
\end{aligned}
$$

where

$$
\begin{aligned}
& x_{1}(k)=\left[X_{11}^{T}(k), X_{12}^{T}(k)\right]^{T}, \\
& x_{2}(k)=\left[X_{21}^{T}(k), X_{22}^{T}(k)\right]^{T}, \\
& u_{1}(k)=\left[U_{11}^{T}(k), U_{12}^{T}(k)\right]^{T}, \\
& u_{2}(k)=\left[U_{21}^{T}(k), U_{22}^{T}(k)\right]^{T} .
\end{aligned}
$$

Suppose the packets are transmitted over the communication channel with a periodic communication sequence $[1,1,2,2]$ in turn, the system equations will evolve according to

$$
\begin{aligned}
x_{1}(k+1)= & A_{11} x_{1}(k)+A_{12} x_{2}(k) \\
& +B_{11}\left[U_{11}^{T}(k), \bar{U}_{12}^{T}(k-1)\right]^{T} \\
& +B_{12}\left[\bar{U}_{21}^{T}(k-1), \bar{U}_{22}^{T}(k-1)\right]^{T} \\
= & A_{11} x_{1}(k)+A_{12} x_{2}(k) \\
& +B_{11}\left[F_{11} X_{11}^{T}(k), F_{11} \bar{X}_{12}^{T}(k-1)\right]^{T} \\
& +B_{12}\left[F_{12} \bar{X}_{21}^{T}(k-1), F_{12} \bar{X}_{22}^{T}(k-1)\right]^{T}, \\
x_{1}(k+2)= & A_{11} x_{1}(k+1)+A_{12} x_{2}(k+1) \\
& +B_{11}\left[U_{11}^{T}(k), U_{12}^{T}(k+1)\right]^{T} \\
& +B_{12}\left[\bar{U}_{21}^{T}(k-1), \bar{U}_{22}^{T}(k-1)\right]^{T}
\end{aligned}
$$

$$
\begin{aligned}
= & A_{11} x_{1}(k+1)+A_{12} x_{2}(k+1) \\
& +B_{11}\left[F_{11} X_{11}^{T}(k), F_{11} X_{12}^{T}(k+1)\right]^{T} \\
& +B_{12}\left[F_{12} \bar{X}_{21}^{T}(k-1), F_{12} \bar{X}_{22}^{T}(k-1)\right]^{T}, \\
x_{1}(k+3)= & A_{11} x_{1}(k+2)+A_{12} x_{2}(k+2) \\
& +B_{11}\left[U_{11}^{T}(k), U_{12}^{T}(k+1)\right]^{T} \\
& +B_{12}\left[U_{21}^{T}(k+2), \bar{U}_{22}^{T}(k-1)\right]^{T} \\
= & A_{11} x_{1}(k+2)+A_{12} x_{2}(k+2) \\
& +B_{11}\left[F_{11} X_{11}^{T}(k), F_{11} X_{12}^{T}(k+1)\right]^{T} \\
& +B_{12}\left[F_{12} X_{21}^{T}(k+2), F_{12} \bar{X}_{22}^{T}(k-1)\right]^{T},
\end{aligned}
$$$$
x_{1}(k+4)=A_{11} x_{1}(k+3)+A_{12} x_{2}(k+3)
$$$$
+B_{11}\left[U_{11}^{T}(k), U_{12}^{T}(k+1)\right]^{T}
$$$$
+B_{12}\left[U_{21}^{T}(k+2), U_{22}^{T}(k+3)\right]^{T}
$$$$
=A_{11} x_{1}(k+3)+A_{12} x_{2}(k+3)
$$$$
+B_{11}\left[F_{11} X_{11}^{T}(k), F_{11} X_{12}^{T}(k+1)\right]^{T}
$$$$
+B_{12}\left[F_{12} X_{21}^{T}(k+2), F_{12} X_{22}^{T}(k+3)\right]^{T},
$$$$
x_{1}(k+5)=A_{11} x_{1}(k+4)+A_{12} x_{2}(k+4)
$$$$
+B_{11}\left[U_{11}^{T}(k+4), U_{12}^{T}(k+1)\right]^{T}
$$$$
+B_{12}\left[U_{21}^{T}(k+2), U_{22}^{T}(k+3)\right]^{T}
$$$$
=A_{11} x_{1}(k+4)+A_{12} x_{2}(k+4)
$$$$
+B_{11}\left[F_{11} X_{11}^{T}(k+4), F_{11} X_{12}^{T}(k+1)\right]^{T}
$$$$
+B_{12}\left[F_{12} X_{21}^{T}(k+2), F_{12} X_{22}^{T}(k+3)\right]^{T},
$$$$
\vdots
$$$$
x_{2}(k+1)=A_{21} x_{1}(k+1)+A_{22} x_{2}(k+1)
$$$$
+B_{21}\left[U_{11}^{T}(k), \bar{U}_{12}^{T}(k+1)\right]^{T}
$$$$
+B_{22}\left[\bar{U}_{21}^{T}(k-1), \bar{U}_{22}^{T}(k-1)\right]^{T} \text {, }
$$ 


$$
\begin{aligned}
& =A_{21} x_{1}(k)+A_{22} x_{2}(k) \\
& +B_{21}\left[F_{21} X_{11}^{T}(k), F_{21} \bar{X}_{12}^{T}(k-1)\right]^{T} \\
& +B_{22}\left[F_{22} \bar{X}_{21}^{T}(k-1), F_{22} \bar{X}_{22}^{T}(k-1)\right]^{T}, \\
& x_{2}(k+2)=A_{21} x_{1}(k+1)+A_{22} x_{2}(k+1) \\
& +B_{21}\left[U_{11}^{T}(k), U_{12}^{T}(k+1)\right]^{T} \\
& +B_{22}\left[\bar{U}_{21}^{T}(k-1), \bar{U}_{22}^{T}(k-1)\right]^{T} \\
& =A_{21} x_{1}(k+1)+A_{22} x_{2}(k+1) \\
& +B_{21}\left[F_{21} X_{11}^{T}(k), F_{21} X_{12}^{T}(k+1)\right]^{T} \\
& +B_{22}\left[F_{22} \bar{X}_{21}^{T}(k-1), F_{22} \bar{X}_{22}^{T}(k-1)\right]^{T}, \\
& x_{2}(k+3)=A_{21} x_{1}(k+2)+A_{22} x_{2}(k+2) \\
& +B_{21}\left[U_{11}^{T}(k), U_{12}^{T}(k+1)\right]^{T} \\
& +B_{22}\left[U_{21}^{T}(k+2), \bar{U}_{22}^{T}(k-1)\right]^{T} \\
& =A_{21} x_{1}(k+2)+A_{22} x_{2}(k+2) \\
& +B_{21}\left[F_{21} X_{11}^{T}(k), F_{21} X_{12}^{T}(k+1)\right]^{T} \\
& +B_{22}\left[F_{22} X_{21}^{T}(k+2), F_{22} \bar{X}_{22}^{T}(k-1)\right]^{T}, \\
& x_{2}(k+4)=A_{21} x_{1}(k+3)+A_{22} x_{2}(k+3) \\
& +B_{21}\left[U_{11}^{T}(k), U_{12}^{T}(k+1)\right]^{T} \\
& +B_{22}\left[U_{21}^{T}(k+2), U_{22}^{T}(k+3)\right]^{T} \\
& =A_{21} x_{1}(k+3)+A_{22} x_{2}(k+3) \\
& +B_{21}\left[F_{21} X_{11}^{T}(k), F_{21} X_{12}^{T}(k+1)\right]^{T} \\
& +B_{22}\left[F_{22} X_{21}^{T}(k+2), F_{22} X_{22}^{T}(k+3)\right]^{T}, \\
& x_{2}(k+5)=A_{21} x_{1}(k+4)+A_{22} x_{2}(k+4) \\
& +B_{21}\left[U_{11}^{T}(k+4), U_{12}^{T}(k+1)\right]^{T} \\
& +B_{22}\left[U_{21}^{T}(k+2), U_{22}^{T}(k+3)\right]^{T} \\
& =A_{21} x_{1}(k+4)+A_{22} x_{2}(k+4) \\
& +B_{21}\left[F_{21} X_{11}^{T}(k+4), F_{21} X_{12}^{T}(k+1)\right]^{T} \\
& +B_{22}\left[F_{22} X_{21}^{T}(k+2), F_{22} X_{22}^{T}(k+3)\right]^{T} \text {. }
\end{aligned}
$$

Using the definition of the buffered states (10), we still obtain the evolution equation (13).

2.2. The General Case. Similarly, if the states of each subsystem are split into $d$ packets and the packets are transmitted in a periodic pattern, we can obtain the general case.

Suppose $\tilde{x}=\left[\hat{x}_{1}^{T} \cdots \widehat{x}_{n}^{T}\right]^{T}, \hat{x}_{i}$ contains past state values for each step in the communication sequence and $\widehat{x}_{i}=$ $\left[x_{i}^{T}(k-p+1) \cdots x_{i}^{T}(k)\right]^{T}$ and $p$ is the length of communication sequence. To describe the general case, we define an integer matrix, $T$, that contains an update sequence for each subsystem's packet. The matrix $T$ will have $n$ rows (one for each subsystem) and $p$ columns. The $(i, j)$ th entry of $T$ will denote the number of steps from the $j$ th step of the communication sequence to the next communication with the $i$ th subsystem. Define $\widetilde{A}_{i j}=J_{p} \otimes I+\sum_{j=1}^{n}\left(E_{n, n} \otimes A_{i j}\right)$, $\Delta \widetilde{A}_{i j}=\sum_{j=1}^{n} E_{n, n} \otimes \Delta A_{i j}$, where $J_{p}$ is the $p \times p$ Jordan matrix composed of all zeros except for ones on the superdiagonal; $E_{i, j}$ is a matrix of zeros with a one in the $(i, j)$ th position. Define

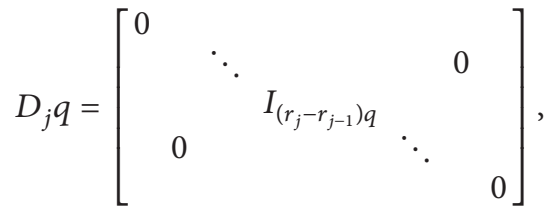

$\widetilde{F}_{i j q}=E_{n, T_{j q}} \otimes B_{i j} F_{i j} D_{j q}$, and $\Delta \widetilde{F}_{i j q}=E_{n, T_{j q}} \otimes \Delta B_{i j} F_{i j} D_{j q}$, which is related to the feedback gains for subsystem $i$ at the $q$ th step in the communication sequence. Now, at each sequence step, $q=1, \ldots, p$, we can write the evolution of all subsystems as

$$
\tilde{x}(k+1)=M_{q} \tilde{x}(k), \quad k \in\{n p+q\},
$$

where

$$
\begin{aligned}
M_{q}= & {\left[\begin{array}{ccc}
\widetilde{A}_{11} & \cdots & \widetilde{A}_{1 n} \\
\vdots & \ddots & \vdots \\
\widetilde{A}_{n 1} & \cdots & \widetilde{A}_{n n}
\end{array}\right]+\left[\begin{array}{ccc}
\Delta \widetilde{A}_{11} & \cdots & \Delta \widetilde{A}_{1 n} \\
\vdots & \ddots & \vdots \\
\Delta \widetilde{\widetilde{A}}_{n 1} & \cdots & \Delta \widetilde{\widetilde{A}}_{n n}
\end{array}\right] } \\
+ & {\left[\begin{array}{ccc}
\widetilde{F}_{11 q} & \cdots & \widetilde{F}_{1 n q} \\
\vdots & \ddots & \vdots \\
\widetilde{F}_{n 1 q} & \cdots & \widetilde{F}_{n n q}
\end{array}\right]+\left[\begin{array}{ccc}
\Delta \widetilde{F}_{11 q} & \cdots & \Delta \widetilde{F}_{1 n q} \\
\vdots & \ddots & \vdots \\
\Delta \widetilde{F}_{n 1 q} & \cdots & \Delta \widetilde{F}_{n n q}
\end{array}\right] . }
\end{aligned}
$$

Remark 2. This paper models uncertain coupled NCSs with multiple packet transmission as switched system. The transmission pattern of the NCSs is closely related to the switching law of the switched system. This switched model not only enables us to design the controller for the NCSs, but also makes it more convenient to deal with the uncertain part.

\section{Stability Analysis and Stabilization Result}

We consider the case that in which all nodes transmitted in a toking-bus. With the toking bus protocol applied, the nodes 
are arranged logically into a ring and transmit their packets in a predetermined order. It can be seen that system (24) is a switched linear system switching among the following subsystems:

$$
\left\{M_{1}, \ldots, M_{p}\right\}
$$

in a periodic manner. Clearly, the original system (1) is stable if the switched system (24) is stable.

The following result gives a sufficient condition on the stability of the NCS (1) with packets in different network channels transmitted in a periodic manner.

Lemma 3 (see [19]). Let the states of multiple NCSs (1) be split into multiple data packets and suppose the transmission of these data packets is in a periodic manner. Then NCS (1) is uniformly asymptotically stable if all the eigenvalues of $\Psi$ are contained within the unit circle; that is, $\left|\lambda_{i}(\Psi)\right|<1$ for $i=1,2, \ldots, n$, where $\Psi=\prod_{i=1}^{i=p} M_{i}$.

The following lemma will play a key rule to design the feedback gain for NCS (1).

Lemma 4 (see [38]). Let the matrices $U, W$, and $\Phi=\Phi^{*}$ be given. Then the following statements are equivalent.

(i) There exists a matrix $V$ satisfying

$$
U V W+(U V W)^{*}+\Phi<0
$$

(ii) The following two conditions hold:

$$
\begin{array}{ccc}
N_{u} \Phi N_{u}^{*}<0 & \text { or } & U U^{*}>0, \\
N_{w}^{*} \Phi N_{w}<0 & \text { or } & W^{*} W>0,
\end{array}
$$

where $N_{u}$ and $N_{w}^{*}$ are, respectively, orthogonal complements of $N$ and $W^{*}$; that is,

$$
N_{u} U=0, \quad N_{w}^{*} W^{*}=0 .
$$

The following lemma will be used to deal with the uncertain part of the NCSs.

Lemma 5 (see [17]). $M, N$, and $\Lambda$ are real matrices with proper dimensions which satisfy $\Lambda^{T} \Lambda \leq I$; then for any positive scalar $\epsilon$, we can get the following inequality:

$$
M \Lambda N+N^{T} \Lambda^{T} M^{T} \leq \varepsilon M M^{T}+\varepsilon^{-1} N^{T} N
$$

We give the stabilization result in the following.
Theorem 6. If there exist a positive definite matrix $P$, matrices $K_{i j}, Y_{i j}$, and positive scalars $\epsilon, \varepsilon_{i}$ satisfying

$$
P B_{i j}=B_{i j} K_{i j}
$$

and the following LMI

$$
\left[\begin{array}{cccccccc}
\Omega & \overline{\bar{M}} & \overline{\bar{N}}^{T} & \overline{\bar{M}_{1}} & {\overline{\overline{N_{1}}}}^{T} & \cdots & {\overline{\overline{M_{n}}}} & {\overline{\overline{N_{n}}}}^{T} \\
\overline{\bar{M}}^{T} & -\Omega_{0} & 0 & 0 & 0 & \cdots & 0 & 0 \\
\overline{\bar{N}} & 0 & -\Omega_{0} & 0 & 0 & \cdots & 0 & 0 \\
{\overline{\overline{M_{1}}}}^{T} & 0 & 0 & -\Omega_{1} & 0 & \cdots & 0 & 0 \\
\overline{\bar{N}_{1}} & 0 & 0 & 0 & -\Omega_{1} & \cdots & 0 & 0 \\
\vdots & \vdots & \vdots & \vdots & \vdots & \ddots & \vdots & \vdots \\
{\overline{\overline{M_{n}}}}^{T} & 0 & 0 & 0 & 0 & \cdots & -\Omega_{n} & 0 \\
\overline{\bar{N}_{n}} & 0 & 0 & 0 & 0 & \cdots & 0 & -\Omega_{n}
\end{array}\right]<0,
$$

where

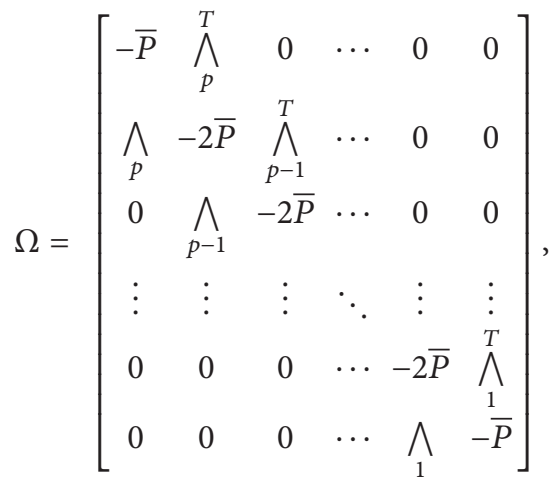

$$
\begin{aligned}
& \bar{P}=\left[\begin{array}{lll}
P & & 0 \\
& \ddots & \\
0 & & P
\end{array}\right] \text {, } \\
& \bigwedge_{q}=\left[\begin{array}{ccc}
P \widetilde{A}_{11} & \cdots & P \widetilde{A}_{1 n} \\
\vdots & \ddots & \vdots \\
P \tilde{\widetilde{A}}_{n 1} & \cdots & P \tilde{\widetilde{A}}_{n n}
\end{array}\right] \\
& +\left[\begin{array}{ccc}
E_{n T_{1 t_{q}}} \otimes B_{11} Y_{11} D_{1 t_{q}} & \cdots & E_{n T_{n t_{q}}} \otimes B_{1 n} Y_{1 n} D_{n t_{q}} \\
\vdots & \ddots & \vdots \\
E_{n T_{1 t_{q}}} \otimes B_{n 1} Y_{n 1} D_{1 t_{q}} & \cdots & E_{n T_{n t_{q}}} \otimes B_{n n} Y_{n n} D_{n t_{q}}
\end{array}\right], \\
& \overline{\bar{M}}=\left[\begin{array}{cccc}
0 & 0 & \cdots & 0 \\
0 & M & \cdots & 0 \\
\vdots & \vdots & \ddots & \vdots \\
0 & 0 & \cdots & M
\end{array}\right]
\end{aligned}
$$




$$
\begin{aligned}
& M=\left[\begin{array}{ccc}
P E_{n, n} \otimes \bar{E}_{11} & \cdots & P E_{n, n} \otimes \bar{E}_{1 n} \\
\vdots & \ddots & \vdots \\
P E_{n, n} \otimes \bar{E}_{n 1} & \cdots & P E_{n, n} \otimes \bar{E}_{n n}
\end{array}\right], \\
& \overline{\bar{N}}=\left[\begin{array}{cccccc}
0 & 0 & 0 & \cdots & 0 & 0 \\
\epsilon N & 0 & 0 & \cdots & 0 & 0 \\
0 & \epsilon N & 0 & \cdots & 0 & 0 \\
\vdots & \vdots & \vdots & \ddots & \vdots & \vdots \\
0 & 0 & 0 & \cdots & 0 & 0 \\
0 & 0 & 0 & \cdots & \epsilon N & 0
\end{array}\right], \\
& N=\left[\begin{array}{ccc}
E_{n, n} \otimes \bar{G}_{1} & \cdots & 0 \\
\vdots & \ddots & \vdots \\
0 & \cdots & E_{n, n} \otimes \bar{G}_{n}
\end{array}\right], \\
& \overline{\overline{M_{i}}}=\left[\begin{array}{cccccc}
0 & 0 & 0 & \cdots & 0 & 0 \\
0 & \varepsilon_{i} M_{i p} & 0 & \cdots & 0 & 0 \\
0 & 0 & \varepsilon_{i} M_{i(p-1)} & \cdots & 0 & 0 \\
\vdots & \vdots & \vdots & \ddots & \vdots & \vdots \\
0 & 0 & 0 & \cdots & \varepsilon_{i} M_{i 2} & 0 \\
0 & 0 & 0 & \cdots & 0 & \varepsilon_{i} M_{i 1}
\end{array}\right] \text {, } \\
& M_{i q}=\left[\begin{array}{ccc}
0 & \cdots & 0 \\
\vdots & & \vdots \\
E_{n T_{1 t_{q}}} \otimes \bar{E}_{i 1} & \cdots & E_{n T_{n t_{q}}} \otimes \bar{E}_{i n} \\
\vdots & & \vdots \\
0 & \cdots & 0
\end{array}\right] \text {, } \\
& \overline{\overline{N_{i}}}=\left[\begin{array}{cccccc}
0 & 0 & 0 & \cdots & 0 & 0 \\
N_{i p} & 0 & 0 & \cdots & 0 & 0 \\
0 & N_{i(p-1)} & 0 & \cdots & 0 & 0 \\
\vdots & \vdots & \vdots & \ddots & \vdots & \vdots \\
0 & 0 & 0 & \cdots & 0 & 0 \\
0 & 0 & 0 & \cdots & N_{i 1} & 0
\end{array}\right], \\
& N_{\text {iq }}
\end{aligned}
$$$$
=\left[\begin{array}{ccc}
E_{T_{1 t_{q} T_{1 t_{q}}} \otimes \bar{H}_{1} Y_{i 1} D_{1 t_{q}}} \cdots & 0 \\
\vdots & \ddots & \vdots \\
0 & \cdots & E_{T_{n t_{q} T_{n t_{q}}}} \otimes \bar{H}_{n} Y_{i n} D_{n t_{q}}
\end{array}\right],
$$$$
\bar{E}_{i j}=\left[\begin{array}{ll}
E_{i j} & 0
\end{array}\right], \quad \bar{G}_{j}=\left[\begin{array}{c}
G_{j} \\
0
\end{array}\right], \quad \bar{H}_{j}=\left[\begin{array}{c}
H_{j} \\
0
\end{array}\right],
$$$$
\Omega_{0}=\operatorname{diag}\{\epsilon I \cdots \epsilon I\}, \quad \Omega_{j}=\operatorname{diag}\left\{\epsilon_{i} I \cdots \epsilon_{i} I\right\} \text {, }
$$

where $q=1, \ldots, p, j=1,2, \ldots, n$, and $i=1,2, \ldots, n$, then multiple NCSs (1) can be robustly stabilized with the state feedback gain

$$
F_{i j}=K_{i j}^{-1} Y_{i j}
$$

Proof. From Theorem 6, the stabilization problem is to compute the feedback gain $F_{i j}$ such that $\Psi$ is Schur-stable. In the Lyapunov framework, the Schur-stability of matrix $\Psi$ can be guaranteed by the existence of a symmetric positive definite matrix $\bar{P}$ such that the following inequality holds:

$$
-\bar{P}+\Psi^{T} \bar{P} \Psi<0
$$

Condition (35) can be written as

$$
\left[\begin{array}{ll}
I & M_{p}^{T}
\end{array}\right]\left[\begin{array}{cc}
-\bar{P} & 0 \\
0 & \Pi_{p-1}^{T} \bar{P} \Pi_{p-1}
\end{array}\right]\left[\begin{array}{c}
I \\
M_{p}
\end{array}\right]<0
$$

where

$$
\Pi_{p-1}=M_{1} M_{2} \cdots M_{p-1} .
$$

We define $N_{u}=\left[\begin{array}{ll}I & M_{p}^{T}\end{array}\right], V=\bar{P}$, and $W=\left[\begin{array}{ll}0 & I\end{array}\right]$. Using Lemma $4,(36)$ is equivalent to the existence of symmetric positive definite matrix $\bar{P}$ such that the following inequality holds:

$$
\begin{aligned}
& {\left[\begin{array}{cc}
-\bar{P} & 0 \\
0 & \Pi_{p-1}^{T} \bar{P} \Pi_{p-1}
\end{array}\right]+\left[\begin{array}{c}
M_{p}^{T} \\
-I
\end{array}\right] \bar{P}\left[\begin{array}{ll}
0 & I
\end{array}\right]} \\
& \quad+\left[\begin{array}{l}
0 \\
I
\end{array}\right] \bar{P}\left[M_{p}-I\right]<0 .
\end{aligned}
$$

Rearranging it, we obtain

$$
\left[\begin{array}{cc}
-\bar{P} & M_{p}^{T} \bar{P} \\
\bar{P} M_{p} & -2 \bar{P}+\Pi_{p-1}^{T} \bar{P} \Pi_{p-1}
\end{array}\right]<0,
$$

which can be written as

$$
\begin{aligned}
& {\left[\begin{array}{ccc}
I & 0 & 0 \\
0 & I & M_{p-1}^{T}
\end{array}\right]\left[\begin{array}{ccc}
-\bar{P} & M_{p}^{T} \bar{P} & 0 \\
\bar{P} M_{p} & -2 \bar{P} & 0 \\
0 & 0 & \Pi_{p-2}^{T} \frac{0}{P} \Pi_{p-2}
\end{array}\right]} \\
& \quad \times\left[\begin{array}{cc}
I & 0 \\
0 & I \\
0 & M_{p-1}
\end{array}\right]<0,
\end{aligned}
$$


where

$$
\Pi_{p-2}=M_{1} M_{2} \cdots M_{p-2}
$$

Repeating this procedure, we can show that (36) can be guaranteed by the following inequality:

$$
\left[\begin{array}{cccccc}
-\bar{P} & M_{p}^{T} \bar{P} & 0 & \cdots & 0 & 0 \\
\bar{P} M_{p} & -2 \bar{P} & M_{p-1}^{T} \bar{P} & \cdots & 0 & 0 \\
0 & \bar{P} M_{p-1} & -2 \bar{P} & \cdots & 0 & 0 \\
\vdots & \vdots & \vdots & \ddots & \vdots & \vdots \\
0 & 0 & 0 & \cdots & -2 \bar{P} & M_{1}^{T} \bar{P} \\
0 & 0 & 0 & \cdots & \bar{P} M_{1} & -\bar{P}
\end{array}\right]<0 .
$$

Let $Y_{i j}=K_{i j} F_{i j}$. Using the definition of $M_{q}(i=1, \ldots, p)$, together with (31), we know (42) is equivalent to

$$
\left[\begin{array}{ccccccc}
-\bar{P} & \bigwedge_{p}^{T}+\Delta \bigwedge_{p}^{T} & 0 & \cdots & 0 & 0 \\
\bigwedge_{p}+\Delta \bigwedge_{p} & -2 \bar{P} & \bigwedge_{p-1}^{T}+\Delta \bigwedge_{p-1}^{T} & \cdots & 0 & 0 \\
0 & \bigwedge_{p-1}+\Delta \bigwedge_{p-1} & -2 \bar{P} & \cdots & 0 & 0 \\
\vdots & \vdots & \vdots & \ddots & \vdots & \vdots \\
0 & 0 & 0 & \cdots & -2 \bar{P} & \bigwedge_{1}^{T}+\Delta \bigwedge_{1}^{T} \\
0 & 0 & 0 & \cdots & \bigwedge_{1}+\Delta \bigwedge_{1} & -\bar{P}
\end{array}\right]
$$

which can be written as

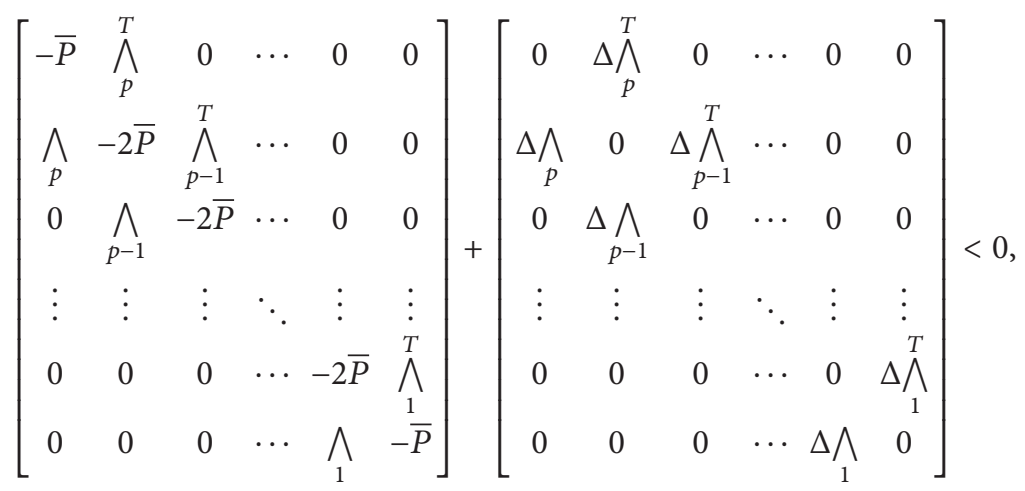

where

$$
\begin{aligned}
& \Delta \bigwedge_{q}=\left[\begin{array}{ccc}
P E_{n, n} \otimes \bar{E}_{11} & \cdots & P E_{n, n} \otimes \bar{E}_{1 n} \\
\vdots & \ddots & \vdots \\
P E_{n, n} \otimes \bar{E}_{n 1} & \cdots & P E_{n, n} \otimes \bar{E}_{n n}
\end{array}\right]\left[\begin{array}{ccc}
E_{n, n} \otimes \bar{\Gamma}_{1} & \cdots & 0 \\
\vdots & \ddots & \vdots \\
0 & \cdots & E_{n, n} \otimes \bar{\Gamma}_{n}
\end{array}\right]\left[\begin{array}{ccc}
E_{n, n} \otimes \bar{G}_{1} & \cdots & 0 \\
\vdots & \ddots & \vdots \\
0 & \cdots & E_{n, n} \otimes \bar{G}_{n}
\end{array}\right] \\
& +\sum_{i=1}^{n}\left[\begin{array}{ccc} 
& 0 & \\
E_{n T_{1 t_{q}}} \otimes \bar{E}_{i 1} & \cdots & E_{n T_{n t_{q}}} \otimes \bar{E}_{i n} \\
& 0 &
\end{array}\right]\left[\begin{array}{ccc}
E_{T_{1 t_{q} T_{1 t_{q}}} \otimes \bar{\Gamma}_{1}} \cdots & 0 \\
\vdots & \ddots & \vdots \\
0 & \cdots & E_{T_{n t_{q} T_{n t_{q}}}} \otimes \bar{\Gamma}_{n}
\end{array}\right] \\
& \times\left[\begin{array}{ccc}
E_{T_{1 t_{q}} T_{1 t_{q}}} \otimes \bar{H}_{1} Y_{i 1} D_{1 t_{q}} & \cdots & 0 \\
\vdots & \ddots & \vdots \\
0 & \cdots & E_{T_{n t_{q}} T_{n t_{q}}} \otimes \bar{H}_{n} Y_{i n} D_{n t_{q}}
\end{array}\right]
\end{aligned}
$$


Define $\overline{\bar{M}}=\bar{M}, \overline{\bar{N}}=\epsilon \bar{N}, \overline{\overline{M_{i}}}=\varepsilon_{i} \bar{M}_{i}, \overline{\bar{N}_{i}}=\bar{N}_{i}, q=$ $1, \ldots, p$. Then LMIs (44) can be rewritten as

$$
\begin{aligned}
\Omega+ & \bar{M} \overline{\bar{\Gamma}}_{1}(k) \bar{N}+\bar{N}^{T} \overline{\bar{\Gamma}}_{1}(k)^{T} \bar{M}^{T} \\
& +\sum_{i=1}^{n} \bar{M}_{i} \overline{\bar{\Gamma}}_{i}(k) \bar{N}_{i}+\bar{N}_{i}^{T} \overline{\bar{\Gamma}}_{i}(k)^{T} \bar{M}_{i}^{T}<0,
\end{aligned}
$$

where

$$
\begin{aligned}
& \overline{\bar{\Gamma}}_{1}(k)=\operatorname{diag}\left\{\Gamma^{\prime}, \Gamma^{\prime}, \ldots, \Gamma^{\prime}\right\}, \\
& \Gamma^{\prime}=\left[\begin{array}{ccc}
E_{n, n} \otimes \bar{\Gamma}_{1} & \ldots & 0 \\
\vdots & \ddots & \vdots \\
0 & \cdots & E_{n, n} \otimes \bar{\Gamma}_{n}
\end{array}\right], \\
& \bar{\Gamma}_{j}=\operatorname{diag}\{\Gamma(k), \Gamma(k), \ldots, \Gamma(k)\}, \\
& \overline{\bar{\Gamma}}_{2}(k)=\operatorname{diag}\left\{0, \Gamma_{p}^{\prime}(k), \Gamma_{p-1}^{\prime}(k), \ldots, \Gamma_{1}^{\prime}(k)\right\}, \\
& \Gamma_{q}^{\prime}=\left[\begin{array}{ccc}
E_{T_{1 t_{q} T_{1 t_{q}}} \otimes \bar{\Gamma}_{1}} \ldots & 0 \\
\vdots & \ddots & \vdots \\
0 & \cdots & E_{T_{n t_{q}} T_{n t_{q}}} \otimes \bar{\Gamma}_{n}
\end{array}\right] .
\end{aligned}
$$

It can be deduced from Lemma 4 that LMI (46) is satisfied if there exist positive scalars $\varepsilon, \varepsilon_{i}$ satisfying

$$
\Omega+\varepsilon \bar{M} \bar{M}^{T}+\varepsilon^{-1} \bar{N}^{T} \bar{N}+\sum_{i=1}^{n} \varepsilon_{i} \bar{M}_{i} \bar{M}_{i}^{T}+\sum_{i=1}^{n} \varepsilon_{i}^{-1} \bar{N}_{i}^{T} \bar{N}_{i}<0 .
$$

By Lemma 3, it is known inequality (48) is equivalent to inequality (32), and NCS (1) can be robustly stabilized with the state feedback gain

$$
F_{i j}=K_{i j}^{-1} Y_{i j}
$$

Remark 7. It is noted that special structured matrices $D_{j q}$ have been introduced into the closed-loop system to deal with multiple packet transmission and this makes the feedback controller design difficult. To solve this problem, equation constraints have been introduced in Theorem 6 and this brings the conservatism.

\section{A Simulation Example}

In this section, a numerical example is given to demonstrate the effectiveness of our method. We consider the case in which the state of the system is split into two parts and the plant has time-varying norm-bounded parameter uncertainties. The coupled system is given by

$$
\begin{aligned}
x_{1}(k+1)= & \left(A_{11}+E_{11} \Gamma_{1}(k) G_{1}\right) x_{1}(t) \\
& +\left(A_{12}+E_{12} \Gamma_{2}(k) G_{2}\right) x_{2}(t) \\
& +\left(B_{11}+E_{11} \Gamma_{1}(t) H_{1}\right) u_{1}(t) \\
& +\left(B_{12}+E_{12} \Gamma_{2}(t) H_{2}\right) u_{2}(t), \\
x_{2}(k+1)= & \left(A_{21}+E_{21} \Gamma_{1}(k) G_{1}\right) x_{1}(t) \\
& +\left(A_{22}+E_{22} \Gamma_{2}(k) G_{2}\right) x_{2}(t) \\
& +\left(B_{21}+E_{21} \Gamma_{1}(t) H_{1}\right) u_{1}(t) \\
& +\left(B_{22}+E_{22} \Gamma_{2}(t) H_{2}\right) u_{2}(t),
\end{aligned}
$$

where

$$
\begin{aligned}
A_{11} & =\left[\begin{array}{cc}
0.3 & 0 \\
0.4 & 0.4
\end{array}\right], \quad A_{12}=\left[\begin{array}{cc}
0.2 & 0.01 \\
0 & 0.2
\end{array}\right], \\
A_{21} & =\left[\begin{array}{cc}
0.05 & 0 \\
0.3 & 0.3
\end{array}\right], \quad A_{22}=\left[\begin{array}{cc}
0 & 0.05 \\
0.3 & 0.3
\end{array}\right], \\
B_{11} & =\left[\begin{array}{c}
0.3 \\
0.6
\end{array}\right], \quad B_{12}=\left[\begin{array}{l}
0.2 \\
0.5
\end{array}\right], \\
B_{21} & =\left[\begin{array}{c}
0.3 \\
1
\end{array}\right], \quad B_{22}=\left[\begin{array}{l}
0.08 \\
0.02
\end{array}\right], \\
E_{11} & =\left[\begin{array}{c}
0.01 \\
-0.001
\end{array}\right], \quad E_{12}=\left[\begin{array}{c}
0.02 \\
-0.002
\end{array}\right], \\
E_{21} & =\left[\begin{array}{c}
0.002 \\
-0.002
\end{array}\right], \quad E_{22}=\left[\begin{array}{c}
0.001 \\
-0.001
\end{array}\right], \\
G_{1} & =\left[\begin{array}{ll}
0.001 & 0.001
\end{array}\right], \quad G_{2}=[0.0020 .002], \\
H_{1} & =0.001, \quad H_{2}=0.002, \\
\Gamma_{1}(k) & =\sin (20 k), \quad \Gamma_{2}(k)=\sin (50 k) .
\end{aligned}
$$

We will show that the NCSs can be stabilized with only a quarter of the state information transmitted, even including time-varying norm-bounded parameter uncertainties. Suppose the controller pays equal attention to each of the two subsystems and the communication sequence is $[1,1,2,2]$. By solving the LMI in Theorem 6 with LMI toolbox [39], we have

$$
P=\left[\begin{array}{cc}
18.0282 & -0.2108 \\
-0.2108 & 2.1266
\end{array}\right],
$$

$$
\begin{aligned}
& \epsilon=15.6610, \quad \varepsilon_{1}=15.6556, \quad \varepsilon_{2}=15.6592, \\
& F_{11}=\left[\begin{array}{ll}
-0.1137 & 0
\end{array}\right], \quad F_{12}=\left[\begin{array}{ll}
0 & -0.2026
\end{array}\right], \\
& F_{21}=\left[\begin{array}{ll}
-0.027 & 0
\end{array}\right], \quad F_{22}=\left[\begin{array}{ll}
0 & -0.5755
\end{array}\right] \text {. }
\end{aligned}
$$




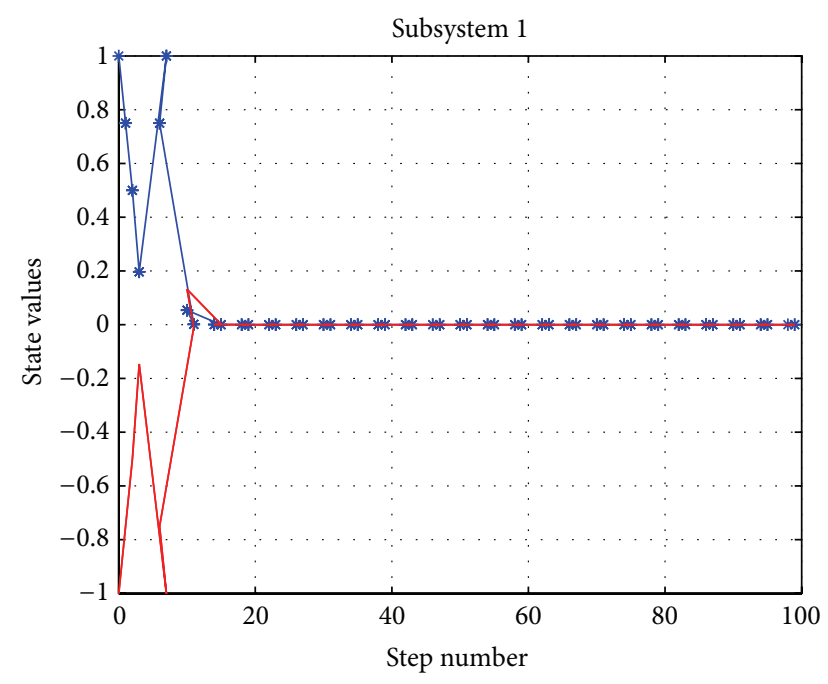

FIGURE 4: The state trajectories of subsystem 1.

With the initial condition $x_{1}(1)=\left[\begin{array}{ll}1 & -1\end{array}\right]^{T}$, $x_{1}(2)=\left[\begin{array}{ll}0.75 & -0.75\end{array}\right]^{T}, x_{1}(3)=\left[\begin{array}{ll}0.5 & -0.5\end{array}\right]^{T}, x_{1}(4)=$ $\left[\begin{array}{ll}0.125 & -0.125\end{array}\right]^{T}, x_{2}(1)=\left[\begin{array}{ll}1 & -1\end{array}\right]^{T}, x_{2}(2)=\left[\begin{array}{ll}0.6 & -0.6\end{array}\right]^{T}$, $x_{2}(3)=\left[\begin{array}{ll}0.4 & -0.4\end{array}\right]^{T}, x_{2}(4)=\left[\begin{array}{ll}0.1 & -0.1\end{array}\right]^{T}$, the state trajectories of two coupled second-order NCSs with multiple packet transmission are shown in Figures 4 and 5. From which we can see that multiple uncertain NCSs (50) with only a quarter of state information transmitted every step can be effectively stabilized with the designed feedback controller. This is a remarkable result, which shows the efficiency of our proposed method. This example illustrates that the switched approach proposed in this paper leads to useful results, because it only requires plant state measurements to be transmitted sparsely. This reduces network traffic without sacrificing stability.

\section{Conclusion}

In this paper, we dealt with robust stabilization of multiple coupled uncertain NCSs with multipacket transmitted over a shared channel. For NCSs acted over a tokingbus, multiple NCSs with multipacket were modeled as a periodically switched system, whose stability guaranteed that of the original system. Sufficient conditions on stability and stabilization of the NCSs with norm-bounded parameter uncertainties were derived. The results obtained here suggest that data packet can be transmitted sparsely to save network bandwidth while preserving the stability of the NCS. This is of practical interest in the application of NCSs.

Future work focuses on studying the case where the communication sequence is stochastic, on one step time delay, and on the real life application.

\section{Conflict of Interests}

The authors declare that there is no conflict of interests regarding the publication of this paper.

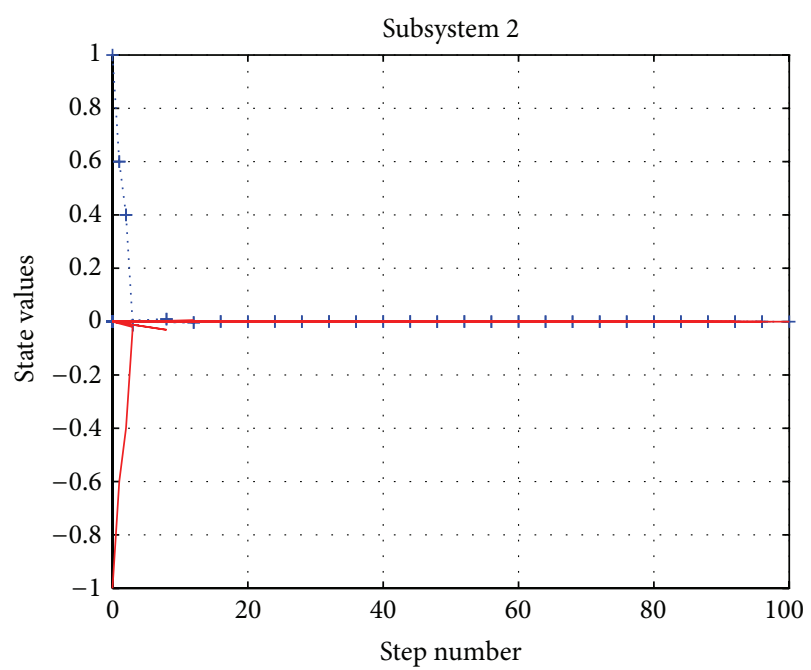

FIgURE 5: The state trajectories of subsystem 2.

\section{Acknowledgments}

This work is supported by the Beijing Municipal Natural Science Foundation (4122075), the National Natural Science Foundation of China under Grants (no. 61004031, no. 61174096, and no. 61104141), the Fundamental Research Funds for the Central Universities, and the Program for New Century Excellent Talents in University.

\section{References}

[1] P. Antsaklis and J. Baillieul, "Special issue on technology of networked control systems," Proceedings of the IEEE, vol. 95, no. 1, pp. 5-8, 2007.

[2] M. C. F. Donkers, W. P. M. H. Heemels, D. Bernardini, A. Bemporad, and V. Shneer, "Stability analysis of stochastic networked control systems," Automatica, vol. 48, no. 5, pp. 917925, 2012.

[3] L. Lu, L. Xie, and M. Fu, "Optimal control of networked systems with limited communication: a combined heuristic and convex optimization approach," in Proceedings of the 42nd IEEE Conference on Decision and Control, pp. 1194-1199, December 2003.

[4] S. Mu, T. Chu, F. Hao, and L. Wang, "Output feedback control of networked control systems," in Proceedings of the IEEE International Conference on Systems, Man \& Cybernetics, pp. 211-216, October 2003.

[5] B. Shen, Z. Wang, and X. Liu, "Sampled-data synchronization control of dynamical networks with stochastic sampling," IEEE Transactions on Automatic Control, vol. 57, no. 10, pp. 26442650, 2012.

[6] M. Darouach and M. Chadli, "The admissibility and control of switched discrete-time singular systems," Systems Science and Control Engineering, vol. 1, no. 1, pp. 43-51, 2013.

[7] D. Ding, Z. Wang, B. Shen, and H. Shu, "the $H_{\infty}$ state estimation for discrete-time complex networks with randomly occurring sensor saturations and randomly varying sensor delays," IEEE Transactions on Neural Networks and Learning Systems, vol. 23, no. 5, pp. 725-736, 2012. 
[8] H. Chan and Ü. Özgüner, "Closed-loop control of systems over a communications network with queues," International Journal of Control, vol. 62, no. 3, pp. 493-510, 1995.

[9] E. O. Elliott, "Estimates of error rates for codes on burst-noise channels," Bell System Technical Journal, vol. 42, no. 5, p. 1977, 1963.

[10] D. Hristu and K. Morgansen, "Limited communication control," Systems \& Control Letters, vol. 37, no. 4, pp. 193-205, 1999.

[11] D. Hristu, "Stabilization of LTI systems with communication constraints," in Proceedings of the American Control Conference, pp. 2342-2346, June 2000.

[12] W. S. Wong and R. W. Brockett, "Systems with finite communication bandwidth constraints. I. State estimation problems," IEEE Transactions on Automatic Control, vol. 42, no. 9, pp. 12941299, 1997.

[13] W. S. Wong and R. W. Brockett, "Systems with finite communication bandwidth constraints. II. Stabilization with limited information feedback," IEEE Transactions on Automatic Control, vol. 44, no. 5, pp. 1049-1053, 1999.

[14] M. Yu, L. Wang, T. Chu, and G. Xie, "Stabilization of networked control systems with data packet dropout and network delays via switching system approach," in Proceedings of the 43rd IEEE Conference on Decision and Control (CDC '04), pp. 3539-3544, December 2004.

[15] M. Yu, L. Wang, T. Chu, and Q. Fu, "Stabilisation of networked control systems with communication constraints," International Journal of Hybrid Systems, vol. 4, pp. 99-112, 2004.

[16] J. Liu, M. Yu, W. Tan, and Z. Chai, "Controller design of multisubsystem with limited communication," in Proceedings of the 29th Chinese Control Conference (CCC '10), pp. 854-858, July 2010.

[17] W. Xiao, M. Yu, and W. Tan, "Controller design for networked control systems with limited bandwidth," in Proceedings of the International Conference on Impulsive Dynamical System Application, pp. 569-573, 2005.

[18] M. Yu, L. Wang, and G. Xie, "A switched system approach to stabilization of networked control systems," Journal of Control Theory and Applications, vol. 4, no. 1, pp. 86-95, 2006.

[19] M. Yu and W. Xiao, "Stabilization of network controlled system with multiple-packet transmission," in Proceedings of the IEEE International Conference on Systems, Man and Cybernetics, pp. 5024-5029, October 2009.

[20] R. Wang, G. P. Liu, W. Wang, D. Rees, and Y. B. Zhao, " $H_{1}$ control for networked predictive control systems based on the switched Lyapunov function method," IEEE Transactions on Industrial Electronics, vol. 57, no. 10, pp. 3565-3571, 2010.

[21] J. Yu and M. Yu, "Multiple controller design for networked control systems with multiple-packet transmissions," in Proceedings of the 24th Chinese Control and Decision Conference (CCDC '12), pp. 1852-1857, 2012.

[22] L. Zhang and D. Hristu-Varsakelis, "Communication and control co-design for networked control systems," Automatica, vol. 42, no. 6, pp. 953-958, 2006.

[23] X. M. Sun, G. P. Liu, D. Rees, and W. Wang, "Stability of systems with controller failure and time-varying delay," IEEE Transactions on Automatic Control, vol. 53, no. 10, pp. 23912396, 2008.

[24] J. Qiu, G. Feng, and J. Yang, "Robust mixed $H_{2} / H_{\infty}$ filtering design for discrete-time switched polytopic linear systems," IET Control Theory \& Applications, vol. 2, no. 5, pp. 420-430, 2008.
[25] J. Qiu, G. Feng, and J. Yang, "New results on robust energyto-peak filtering for discrete-time switched polytopic linear systems with time-varying delay," IET Control Theory \& Applications, vol. 2, no. 9, pp. 795-806, 2008.

[26] Y. Zheng and G. Feng, "Exponential H- $\infty$ filtering for discretetime switched state-delay systems under asynchronous switching," Asian Journal of Control, vol. 13, no. 1, pp. 1-10, 2013.

[27] V. N. Phat, J. Jiang, A. V. Savkin, and I. R. Petersen, "Robust stabilization of linear uncertain discrete-time systems via a limited capacity communication channel," Systems \& Control Letters, vol. 53, no. 5, pp. 347-360, 2004.

[28] D. Wu, J. Wu, and S. Chen, "Robust $H_{\infty}$ control for networked control systems with uncertainties and multiple-packet transmission," IET Control Theory \& Applications, vol. 4, no. 5, pp. 701-709, 2010.

[29] J. Yu, L. Wang, and M. Yu, "A new approach to controller design for networked control systems with multiple-packet transmissions," International Journal on Systems, Control and Communications, pp. 158-177, 2011.

[30] M. C. F. Donkers, W. P. M. H. Heemels, N. van de Wouw, and L. Hetel, "Stability analysis of networked control systems using a switched linear systems approach," IEEE Transactions on Automatic Control, vol. 56, no. 9, pp. 2101-2115, 2011.

[31] L. Greco, A. Chaillet, and A. Bicchi, "Exploiting packet size in uncertain nonlinear networked control systems," Automatica, vol. 48, no. 11, pp. 2801-2811, 2012.

[32] M. Yu, L. Wang, T. Chu, and G. Xie, "Stabilization of a collection of linear systems with limited information," Asian Journal of Control, vol. 9, no. 1, pp. 80-86, 2007.

[33] N. Ding, M. Yu, and W. Xiao, "A switched approach to stabilization of multiple networked control systems," in Proceedings of the 6th IEEE Conference on Industrial Electronics and Applications (ICIEA '11), pp. 1056-1061, June 2011.

[34] S.-L. Dai, H. Lin, and S. S. Ge, "Scheduling-and-control codesign for a collection of networked control systems with uncertain delays," IEEE Transactions on Control Systems Technology, vol. 18, no. 1, pp. 66-78, 2010.

[35] S.-L. Dai, H. Lin, and S. S. Ge, "A switched system approach to scheduling of networked control systems with communication constraints," in Proceedings of the 28th Chinese Decision and Control (CDC '09), pp. 4991-4996, December 2009.

[36] Z. Ji, L. Wang, and X. Guo, "On controllability of switched linear systems," IEEE Transactions on Automatic Control, vol. 53, no. 3, pp. 796-801, 2008.

[37] Z. Ji, H. Lin, G. Feng, and X. Guo, "Controllability structure decomposition for switched linear systems," Transactions of the Institute of Measurement and Control, vol. 32, no. 6, pp. 736-755, 2010.

[38] R. E. Skelton, T. Iwasaki, and K. Grigoriadis, A Unified Approach to Linear Control Design, Taylor and Francis series in Systems and Control, 1997.

[39] S. Boyd, L. El Ghaoui, E. Feron, and V. Balakrishnan, Linear Matrix Inequalities in System and Control Theory, Society for Industrial and Applied Mathematics, Philadelphia, Pa, USA, 1994. 


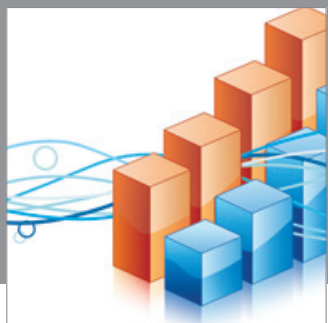

Advances in

Operations Research

mansans

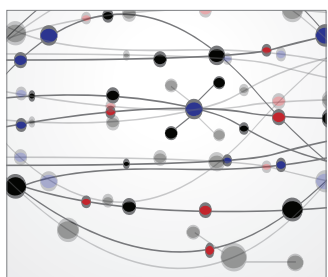

The Scientific World Journal
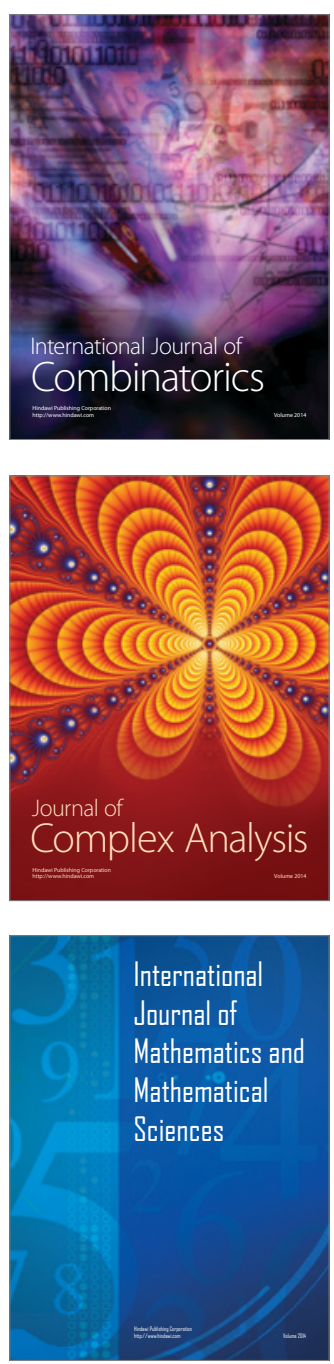
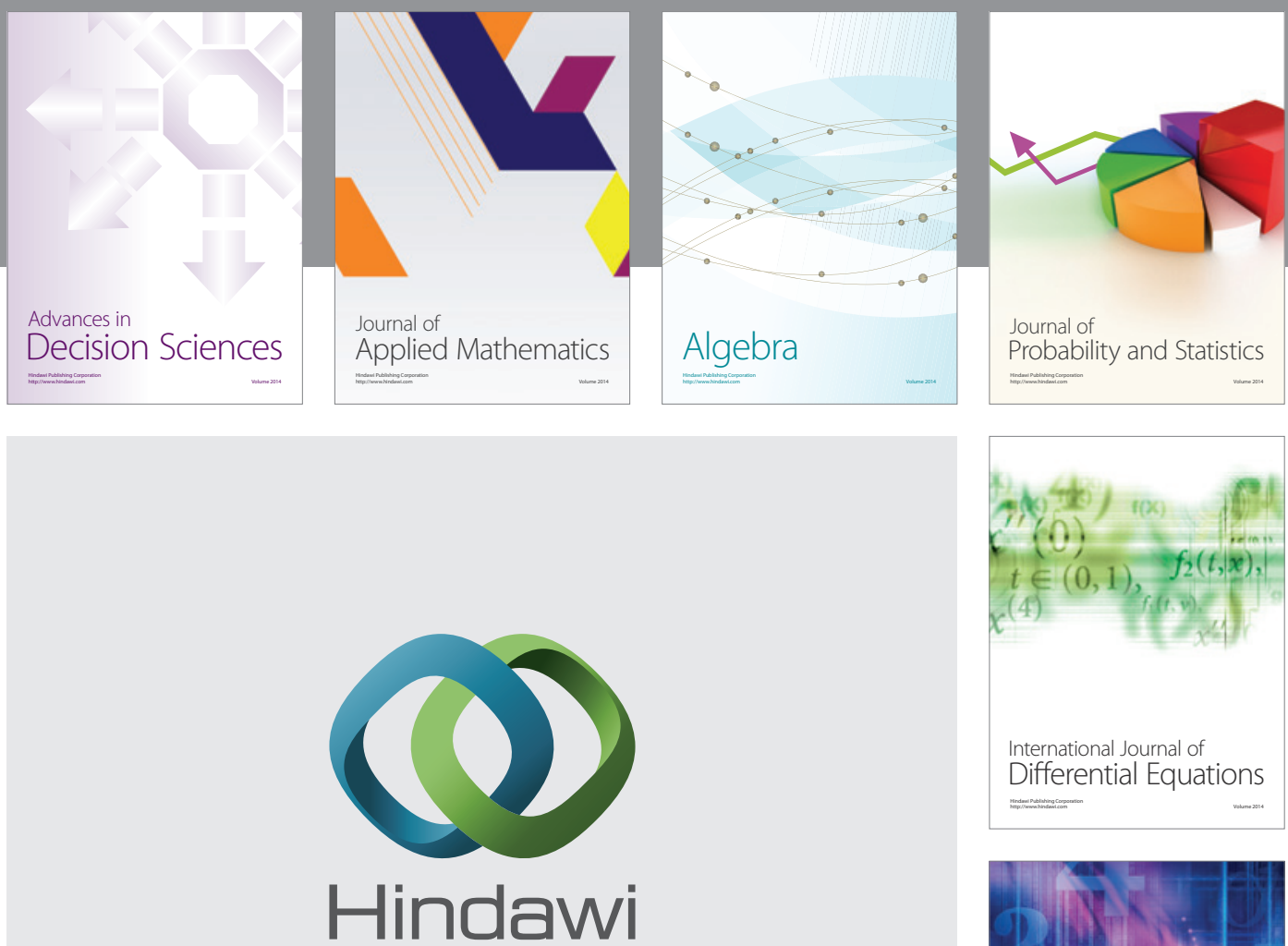

Submit your manuscripts at http://www.hindawi.com
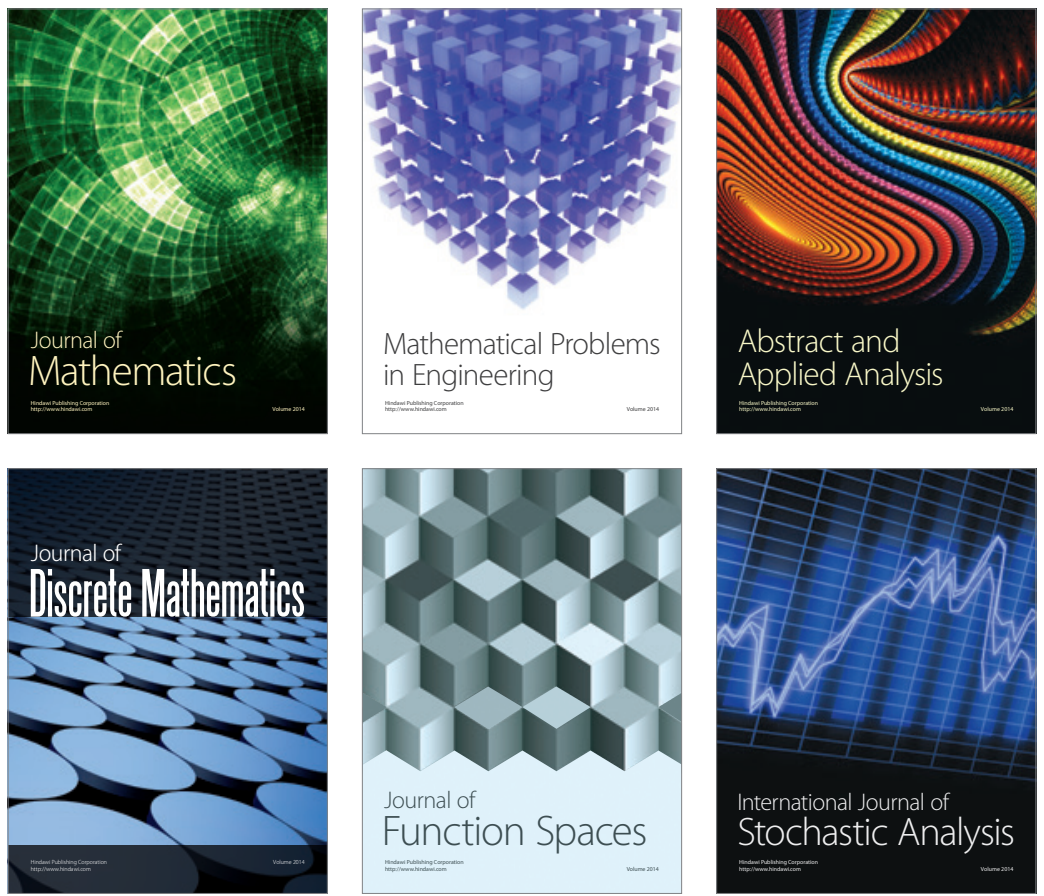

Journal of

Function Spaces

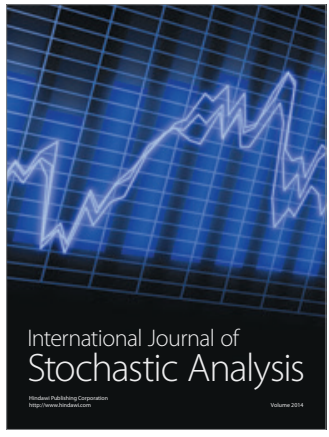

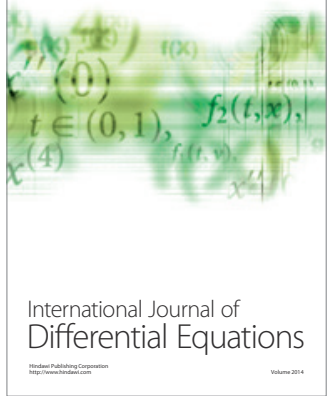
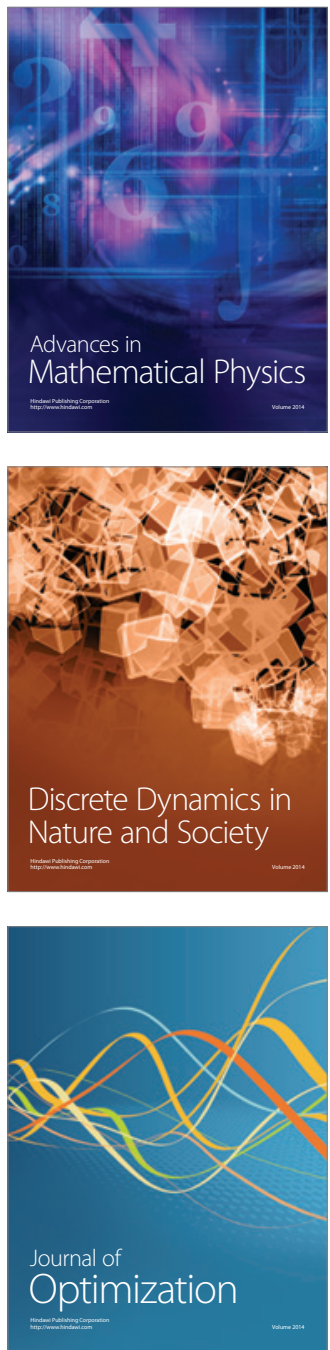